\title{
Magnetovision Scanning System for Detection of Dangerous Objects
}

\author{
M. NOWICKI*, R. SzEWCZYK \\ Institute of Metrology and Biomedical Engineering, Warsaw University of Technology, \\ św. A. Boboli 8, 02-525 Warszawa, Poland
}

\begin{abstract}
This paper presents an application of magnetovision based measurements to develop a method for passive detection of dangerous ferromagnetic objects. Scanning system was designed and built to study the magnetic field vector distributions. The measurements of the Earth's field disturbances caused by ferromagnetic objects were carried out. The ability for passive detection of selected dangerous objects was demonstrated. Further data processing allowed for determining the $(\mathrm{x}, \mathrm{y})$ coordinates of the object relative to the plane of measurement, and even the possibility of calculating the distance from the object. The results obtained indicate that it is possible to detect and determine the location of dangerous ferromagnetic objects. This opens the new way to use magnetovision in public security systems, in particular for the detection of dangerous objects by police and sapper robots.
\end{abstract}

DOI: $10.12693 /$ APhysPolA.126.382

PACS: $81.70 .-\mathrm{q}, 85.75 . \mathrm{Ss}$

\section{Introduction}

Magnetovision utilizes the measurement of the distribution of magnetic field in a particular plane and presenting it with a 2D image. The name "magnetovision" comes from an analogy with thermal imaging. The most suitable sensors for magnetovision are thin-film magnetoresistive sensors. They exhibit high sensitivity and have small size [1]. Resolution of images depends directly on the number of measurement points per meter.

Magnetovision studies, carried out previously, were focused on the ability to measure stress in the ferromagnetic materials, in relation to the inverse magnetostrictive (Villari) effect $[2,3]$. In that case, external magnetic field was not applied. Obtaining magnetovision images of unknown objects from a greater distance and on a larger surface area, required the development of new methods for measuring and processing the results. The application of passive magnetovision system is important, because the active metal detectors can be sensed by the specially constructed detonators. This applies particularly to the new generations of landmines, reacting to the presence of active detectors, which represents a direct threat to sapper's life [4].

\section{Research methodology}

For the purpose of the study an XY scanning system was designed and built, with a single, tri-axial magnetoresistive Honeywell HMR2300 sensor. Unlike existing magneto-vision systems, application of tri-axial sensor enabled gathering information about the magnetic induction vector value and its direction with respect to each measurement point in the scanning plane, not only its absolute value. During the measurements, no additional

*corresponding author; e-mail: m.nowicki@mchtr.pw.edu.pl fields have been applied. Therefore only background disturbances were measured, mainly disturbances of the natural Earth magnetic field. Scanning probe system transit along parallel lines with a given interval, setting the measurement plane.

The simplest solution for separation of distortion generated by a sample object from the background is the measurement without the test object and subtracting the result from the measurement with an object. This method gives the best results, however, it is possible only in certain conditions. For this reason, a method of differential measurement was developed, minimizing the impact of the background on the measurement result, including both the Earth magnetic field as well as the other sources. In its simplest form, a differential measurement is the measurement in two parallel planes, at the height $x$ above the test object, and $x+a$, where $a$ is the distance between the planes of measurement, and subtracting the results. Distribution of flux density lines near a ferromagnetic object placed in the Earth's magnetic field is similar to a permanent magnet field distribution. Induction of the magnetic field on the axis of the magnet, in vacuum, at a distance $x$ from its center is expressed by the formula:

$$
\boldsymbol{B}=\frac{\mu_{0}}{2 \pi x^{3}} \boldsymbol{m}=C \frac{1}{x^{3}},
$$

where $\boldsymbol{m}$ - magnetic dipole moment $\left(\mathrm{A} \mathrm{m}^{2}\right), C=\frac{\mu_{0}}{2 \pi} \boldsymbol{m}$ - induction replacement constant $\left(\mathrm{T} \mathrm{m}^{3}\right)$.

Since the value of the flux density $B$ is reduced in proportion to the distance from the source raised to the power of three (1), it is possible to distinguish between powerful sources located far and weak sources near the plane of measurement, as the near objects give bigger difference of distortion between the measurement planes. Therefore differential bi-plane measurement gives the absolute value of the difference in magnetic induction value between the measurement planes which is sufficient to de- 
tect and localize the hidden object, even a complicated one, from the magnetic modeling point of view.

\section{Results}

Measurements were carried out on a test stand setup described in previous chapter. The testing area of $200 \times 200 \mathrm{~mm}$ was adopted, with 10 parallel measurement lines. On each line there were 100 measurement points. Then the obtained $100 \times 10$ matrix with results was interpolated to $100 \times 100$ points, which allowed for a cleaner picture. On the base of gathered data, the magnetovision image of magnetic field distribution was calculated. Sample used for testing was a steel cylinder, $80 \mathrm{~mm}$ diameter and $20 \mathrm{~mm}$ height, located in the center of measurement plane. Such element can simulate landmine for tests [5]. Distance between measurement planes was set to $a=40 \mathrm{~mm}$.

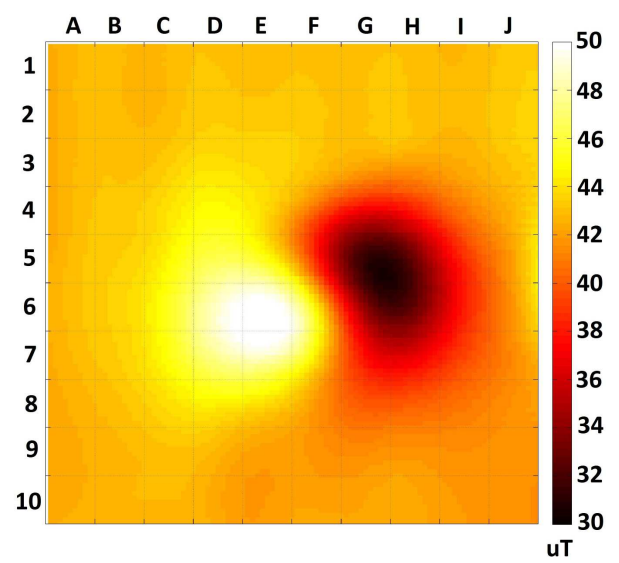

Fig. 1. Results of one-plane measurements, absolute values.

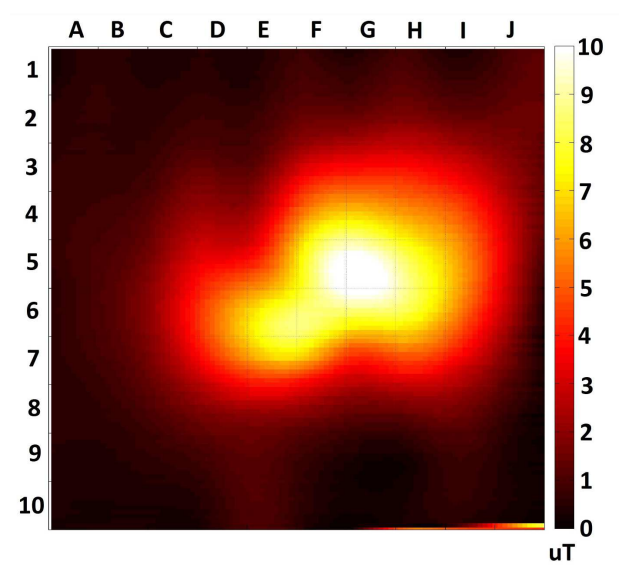

Fig. 2. Results of bi-plane differential measurement.

Figure 1 shows one-plane, absolute values measurement within $80 \mathrm{~mm}$ of the sample. Results of up to $\pm 12 \mu \mathrm{T}$ were obtained for absolute value of the disorders in respect to the background field. Figure 2 shows bi-plane differential measurement within $80 \mathrm{~mm}$ of the same sample. Minimization of the background impact on the result is clearly visible. Figure 3 shows a picture of the three-dimensional distribution of magnetic induction vectors in all measurement points. Absolute values were obtained by differential measurement in a single plane with the influence of the background removed.

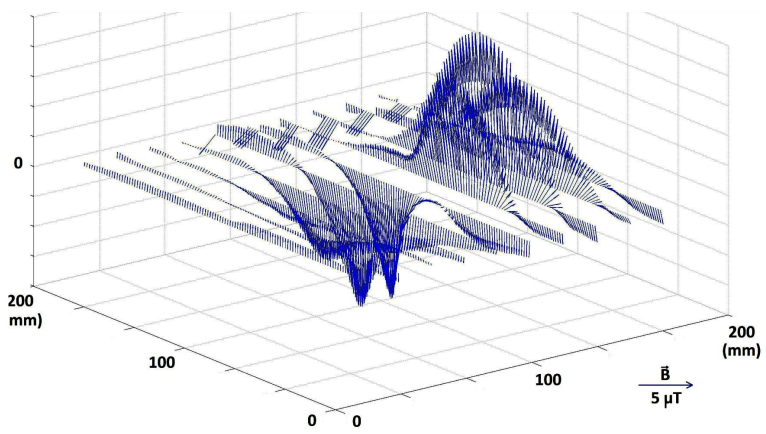

Fig. 3. Bi-plane measurement, magnetic induction vector length and direction visualisation.

\section{Conclusions}

Experimental setup for planar measurements of vector distribution of weak magnetic fields was built. Moreover, new methodology of measurement, leading to decreasing the impact of magnetic background was presented. The developed methods allow visualization of the distribution of the magnetic induction vector absolute values, its gradient as well as the value and direction of the magnetic flux density vector in different measurement points. Obtained results indicate, that it is possible to detect and determine the location of dangerous ferromagnetic objects. This opens the way to use magnetovision in public security systems, in particular for the detection of dangerous objects by police demining robots [6]. Such system can also be used in non-destructive testing, e.g. for detection of structural defects inside railroad tracks.

\section{Acknowledgments}

Work was supported by The National Center for Research and Development, grant no. O ROB 0015 01/ID15/1.

\section{References}

[1] S. Tumański, Thin film magnetoresistive sensors, IOP Publ., Bristol 2001.

[2] J. Kaleta, J. Zebracki, Fatigue Fracture Eng. Mater. Struc. 19, 1435 (1996).

[3] S. Gontarz, S. Radkowski, IEEE Trans. Magn. 48, 1143 (2012).

[4] D. Guelle, A. Smith, A. Lewis, T. Bloodworth, Metal detector handbook for humanitarian demining, Office for Official Publications of the European Communities, Luxembourg 2003.

[5] S.D. Billings, C. Pasion, S. Walker, L. Beran, IEEE Trans. Geosci. Rem. Sens. 44, 2115 (2006).

[6] R. Czupryniak, M. Trojnacki, Journal of Automation, Mobile Robotics and Intelligent Systems 4, 26 (2010). 